\title{
Model for athermal enhancement of molecular mobility in solid polymers by light
}

\author{
Zouheir Sekkat $0^{*}$ \\ Department of Chemistry, Faculty of Sciences, Mohammed V University in Rabat, Rabat 10000, Morocco; \\ Optics and Photonics Center, Moroccan Foundation for Advanced Science, Innovation and Research, Rabat 10100, Morocco; \\ and Department of Applied Physics, Graduate School of Engineering, Osaka University, 2-1 Yamadaoka, Suita, Osaka 565-0871, Japan
}

(Received 10 May 2020; accepted 24 July 2020; published 16 September 2020)

\begin{abstract}
Molecular motion in polymers is frozen below the glass transition temperature $T_{g}$ and changes of viscoelastic functions are most spectacular near $T_{g}$. Exceptional enhancement of molecular mobility and a decrease of polymer viscosity, by several orders of magnitude, down to the viscous flow regime, are observed way below $T_{g}$ by light absorption. Relaxation processes, which take decades to centuries in some high- $T_{g}$ polymers, are reduced to minute timescales by sub- $T_{g}$ light absorption. Here we develop a model for this intriguing albeit spectacular action of light on glass forming materials and we propose experiments to relate light absorption to materials properties. The model provides a solution to a long-lasting problem of how molecular mobility is enhanced in solid polymers by photoisomerization and provides a tool for a better understanding of the relationship between light absorption and material properties and developing photosensitive polymers for light to mechanical energy transduction.
\end{abstract}

DOI: 10.1103/PhysRevE.102.032501

\section{INTRODUCTION}

If a polymer is cooled, from the viscous flow to the glassy regime, its viscosity $\eta$ is dramatically increased by several orders of magnitude, much like a supercooled liquid. Viscosity reflects the underlying movement of the molecules in the polymer, and its variation is proportional to that of the timescale $\tau$ of the relevant relaxation process; therefore, $\tau$ is increased as well, by several orders of magnitudes upon cooling [1]. The reciprocal phenomenon occurs upon heating. Exceptional enhancement of molecular mobility and a reversible change from solid to viscous regimes occur athermally in photoactive polymers when units of the polymer undergo structural rearrangement upon light absorption [2-6]. The photoactive units are smart molecular machines, which are fueled by light, and they switch between isomeric configurations at high frequency (gigahertz) [7], driving polymer chains in motion upon light action, as has been observed in the solid phase in a number of polymers by several researchers [8]. This paper develops a model for light-induced free volume and viscosity change. We demonstrate that the decrease of viscosity and relaxation time in solid films of polymers can be explained by an increase in the free volume of the polymer by light absorption. Upon light absorption, the free volume of the polymer is increased by continuous cyclic isomerization of the photoactive molecules. The isomerization of the azo dyes triggers molecular and segmental motions of their nanocogent environment by pushing the neighboring atoms (Fig. 1). When

\footnotetext{
*z.sekkat@mascir.ma
}

Published by the American Physical Society under the terms of the Creative Commons Attribution 4.0 International license. Further distribution of this work must maintain attribution to the author(s) and the published article's title, journal citation, and DOI. light absorption stops, so do molecular motions and polymer configurations freeze and require heating to near the glass transition temperature $T_{g}$ to induce appreciable motion in the absence of an external stimulus. Stochastic motion, due to thermal fluctuations, is always present in the material.

Azo dyes, for example, disperse red 1 (DR1), exist in two isomeric forms (trans and cis) and they undergo molecular motion by shape changes between these isomers upon light absorption; they constitute a good example of light-fueled molecular machines that act as light to mechanical energy transducers [9]. The dyes, which are embedded into polymers by, for example, chemical attachment, are in perpetual motion during light absorption, i.e., they undergo billions of shape changes per second owing to picosecond isomerization under absorption of light of the visible wavelength, and they cause molecular displacement of the surrounding medium in their nanocogent environment $[10,11]$ and create additional free volume, thereby reducing friction and viscosity and enhancing molecular mobility. For a 532-nm photon, the absorbed energy is $2.331 \mathrm{eV}$ and the $3.5-\AA$ change in length of the long axis of the molecule yields an estimate for the force per molecule in the nanonewton range. Such a force magnitude has been measured in a single polymer chain [12] and in an ensemble of chains in a solid film configuration when a molecule is addressed by a single photon on average [6]. The potential that drives the polymer motion scales with the density $N$ of the active molecules (molecules $\mathrm{cm}^{-3}$ ) and the total energy absorbed by the dyes reads

$$
u=N v\left\{(1-x) \sigma_{T} \phi_{T C}+x \sigma_{C} \phi_{C T}\right\} \mathfrak{s},
$$

where $u$ is the energy dose absorbed by the material (expressed in joules), $\mathfrak{s}=I_{0} t$ is the incident energy dose per unit area (expressed in $\mathrm{J} \mathrm{cm}^{-2}$ ), $I_{0}$ is the incident light intensity (in $\mathrm{mW} \mathrm{cm}^{-2}$ ), $t$ is the time (in seconds), $v$ is the absorption volume (in $\mathrm{cm}^{3}$ ), $\sigma_{T}$ and $\sigma_{C}$ are the isotropic absorption cross sections of the trans and cis isomers (in $\mathrm{cm}^{2}$ molecules ${ }^{-1}$ ), 


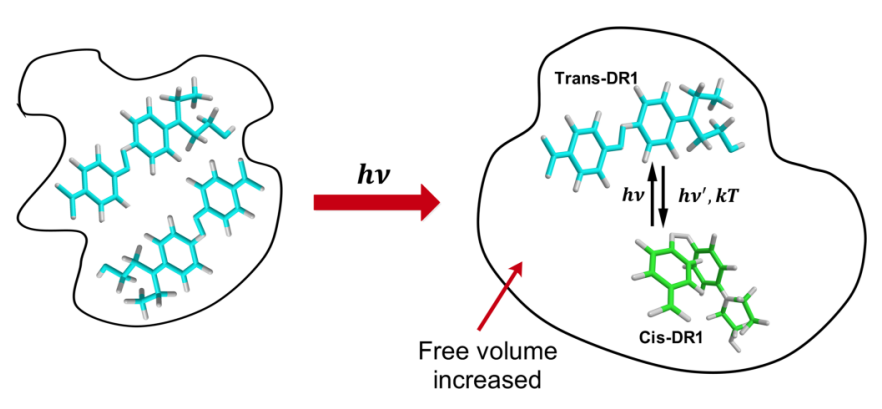

FIG. 1. Schematics of free volume increase in a polymer upon light absorption and of trans $\leftrightarrow$ cis isomerization of an azo dye, e.g. DR1.

respectively, $\phi_{T C}$ and $\phi_{C T}$ are the isomerization quantum yields which represent the probability per absorbed photon for the molecule to undergo isomerization, and $x$ is the extent of the cis isomers. The subscripts $T C$ and $C T$ refer to the trans $\rightarrow$ cis and cis $\rightarrow$ trans isomerization reactions, respectively. If $V$ $(\propto 1 / N)$ is the specific volume and $V_{\infty}$ an occupied volume, including the volume of the molecules and the volumes of their rotational and vibrational motions, the free volume is $V_{f}=V-V_{\infty}$ and the fractional free volume is $f=V_{f} / V_{\infty}$. Here $V_{\infty}$ includes the activation volume necessary for onemolecule isomerization $\Delta V^{\#}$ and the light-created free volume per molecule exceeds $\Delta V^{\#}$. Even though the latter does not directly enter the model (discussed later), we mention it to clearly differentiate it from the free volume created by photoisomerization. Here $\Delta V^{\#}$ is of the same order as the size of the molecule or the repeat unit. For example, $\Delta V^{\#}=(111 \pm$ 7) $\AA^{3}$ was experimentally determined, from photo-orientation experiments at hydrostatic pressure, for DR1 tethered to a polymethyl methacrylate (PMMA) copolymer, with 10\% DR1 per monomer unit (molecular weight $M_{w} \sim 163000 \mathrm{~g} / \mathrm{mol}$ and $T_{g} \sim 123{ }^{\circ} \mathrm{C}$ ), in a $1-\mu \mathrm{m}$ thin solid film configuration having a fractional free volume of $\sim 2.4 \%$. A pressure of $150 \mathrm{MPa}$ at $T_{g}-98^{\circ} \mathrm{C}$ inhibited most of the DR1 dyes to isomerize in this solid film [13]. Many polymers containing photochromic dyes, either as guests or as pendant side groups or even cross-linked, exhibit a fractional free volume in the range of $0.02-0.03$ [14].

\section{MODEL}

\section{A. Light-induced change of free volume and viscosity}

We consider that molecular mobility, under any temperature and pressure conditions, primarily depends on $V_{f}$, and therefore viscosity and relaxation times can be expressed as a function of $V_{f}$ as well. We express the relative elementary increase of the free volume by light absorption, by an expansion coefficient $\gamma_{f}=\left(1 / V_{f}\right)\left(\partial V_{f} / \partial u\right)$ implying an exponential dependence on $u$,

$$
f=f_{r} \exp \left[\gamma_{f}\left(u-u_{r}\right)\right]
$$

In the case of a small relative increase of the free volume upon light absorption $\left(\gamma_{f}\right.$ small $)$, we obtain a linear relation between $f$ and $u$,

$$
f=f_{r}\left[1+\gamma_{f}\left(u-u_{r}\right)\right],
$$

where $f_{r}$ is the fractional free volume at the reference absorbed energy dose $u_{r}$. The material is allowed to expand at constant temperature and pressure. The energy deposited into the material upon light absorption is transformed into mechanical work that drives the rearrangement of matter and expands its volume. Cohen and Turnbull [15] formalized the empirical equation of the viscosity of Doolittle and Doolittle [16] by using the theory of molecular transport in liquids and glasses. They derived the diffusion coefficient $D$ of hard spheres in a liquid and compared it to the Stokes-Einstein (SE) expression for $D$, derived from hydrodynamics for nearly spherical molecules, to derive the relation between the free volume and viscosity $\eta$ [Eq. (4)]. The SE equation is applicable to macromolecules and polymers and therefore it is applicable to azopolymers as well $[6,7,9]$. In the original paper by Cohen and Turnbull, it was assumed that the SE relationship held around $T_{g}$; however, it is well known from the literature that the SE relationship breaks down close to $T_{g}$ in both supercooled liquids and polymer glasses owing to dynamic heterogeneity, i.e., to the presence of particles with significantly different mobility relative to the average mobility [17,18] (while our paper does not intend to resolve this discrepancy in the literature, we thought it is worth mentioning this caveat)

$$
\eta=A \exp \left(B V_{\infty} / V_{f}\right)
$$

where $A$ and $B$ are constants, with $B \cong 1$, and $\eta$ is expressed in Pa s. Equation (4) implies that the free volume is the sole parameter in determining the rates of molecular rearrangements and transport phenomena such as diffusion and viscosity. Inserting Eq. (3) into Eq. (4) yields, after rearrangement,

$$
\ln \left(\eta / \eta_{r}\right)=\frac{-\gamma_{f}\left(u-u_{r}\right) / f_{r}}{1+\gamma_{f}\left(u-u_{r}\right)} .
$$

This equation is the analog of the equation derived by Williams et al., i.e., the Williams-Landel-Ferry (WLF) shift factor, with $u$ being the analog of temperature $T$ [19]. The WLF law is used to rationalize molecular motion in polymers as a function of the difference between the operating temperature $T$ and $T_{g}$, that is, motion in polymers is frozen way below $T_{g}$ and it is strongly enhanced when $T$ approaches $T_{g}$. In our case, and as it can be clearly seen from Eq. (5), motion, represented by viscosity change, is dictated by the energy difference $\Delta u=u-u_{r}$. The dependence of $\ln \left(\eta / \eta_{r}\right)$ as a function of $\Delta u$, which must be linear for $\gamma_{f} \Delta u \ll 1$, could be experimentally verified and $\gamma_{f}$ and $f_{r}$, which are material dependent, could be measured from experiments (discussed later). Here $f_{r}$ is unitless and $\left[\gamma_{f}\right]=1 \mathbf{J}^{-1}$. Next we test the validity of the model. For a small change of $\gamma_{f} \Delta u$ and to stick with the initial assumption of the linearity $f=f_{r}\left(1+\gamma_{f} \Delta u\right)$, we slightly increase the fractional free volume by a few percent, say, $5 \%$, i.e., $f=1.05 f_{r}$, implying that $\gamma_{f} \Delta u=0.05$. This leads to a viscosity decrease by nearly an order of magnitude $\left(\eta / \eta_{r} \sim 0.14\right)$. If the absorbed energy dose is decreased by an order of magnitude, i.e., $\gamma_{f} \Delta u=$ 0.005 , viscosity is still decreased by $\sim 20 \%\left(\eta / \eta_{r} \sim 0.81\right)$. We will go on to show that this situation corresponds to actual experimental observations.

It is remarkable to note that a very small, i.e., a few percent, change in the fractional free volume decreases the viscosity 


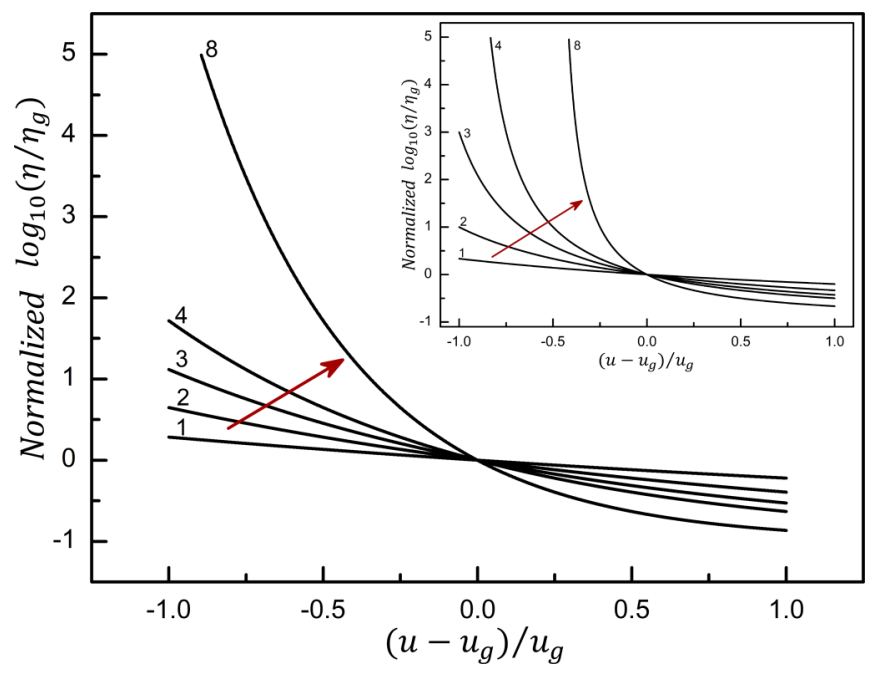

FIG. 2. Plot of $\left(1 / A_{1}\right) \ln \left(\eta / \eta_{r}\right)$ versus $u-u_{r}$ calculated with $A_{1}=1 / 2.303 f_{r} \sim 18.1$ for $f_{r} \sim 2.4 \%$, constant, and normalized to 1 and $A_{2}=\gamma_{f} u_{r}$ a parameter, varied as multiples (1, 2, 3, 4, and 8, as indicated on the curves) of $\gamma_{f} u_{r} \sim 0.25$ for $\gamma_{f}$ given in the text and $u_{r}=u_{g}=37 \mathrm{~J}$ near the glass transition energy of the PMMADR1 sample (see Sec. III). The $A_{1}$ and $A_{2}$ could be extracted from experiments. The $x$ axis is normalized as $\left(u-u_{r}\right) / u_{r}$. In the inset the behavior of viscosity change is accurately described by Eq. (6) and approximated by Eq. (5) (inset) for small $\left(u-u_{r}\right) / u_{r}$; viscosity changes depend strongly on $\gamma_{f}$. Larger $\gamma_{f}$ leads to a larger viscosity change; e.g. $\eta$ may decrease by several decades for large $\gamma_{f}$. The arrow indicates the direction of increase of $\gamma_{f}$. We set $u_{r}=u_{g}$, i.e., the energy that causes the material to move from the glassy to the viscoelastic state; it is interesting to note that viscosity change behaviors are different depending on whether $u_{g}$ is approached from the left, i.e., below $u_{g}$, or from the right, i.e., above $u_{g}$. Indeed, below $u_{g}$, light action is much more effective in the glassy state than above $u_{g}$, where the material is already in the rubbery and viscous flow regime.

by nearly an order of magnitude and a minute change of the fractional free volume decreases appreciably the viscosity. If the fractional free volume increases by more than $5 \%$, the linear model is less accurate (Fig. 2) and the exact formula, i.e., Eq. (2), should be combined with Eq. (4) to yield, after rearrangement,

$$
\ln \left(\eta / \eta_{r}\right)=\left(e^{-\gamma_{f}\left(u-u_{r}\right)}-1\right) / f_{r} .
$$

Increasing the fractional free volume by $20 \%-30 \%$ decreases the viscosity by three to four orders of magnitude (discussed later).

\section{B. Light-induced change in relaxation times}

The viscosity and the time $\tau$ of structural relaxation are proportional to each other; therefore, $\tau$ can replace $\eta$ in Eq. (4) and $A$ should be changed to a different constant $A^{\prime}\left(\tau=A^{\prime} \exp [B / f]\right)$. Thus the dependence of $\ln \left(\tau / \tau_{r}\right)$ on $u$ is obtained by replacing $\eta$ by $\tau$ in Eqs. (5) and (6) for the linear and general cases of the dependence of $f$ on $u$, respectively, and the analysis pertaining to $\eta$ with respect to $u$ holds for $\tau$. That is, a small increase in the fractional free volume considerably decreases $\tau$. Increasing $f$ by $5 \%$ and $30 \%$ decreases $\tau$ by one and four decades, respectively. Orientational order in solid films of high- $T_{g}$ poled polymers takes tens of years to centuries to decay to $1 / e$ from the original order [20-23] and it decays in minutes way below $T_{g}$ when the ordered molecules undergo cyclic isomerization [24-26]. Even though reorientation of the azo dye plays a major role in this depoling phenomenon [24], polymer chain motion occurs during light-induced depoling [25], which must create additional free volume in the polymers studied. An interesting experiment to perform is the study of the relaxation of the polar order of a nonphotochromic probe molecule attached to a light sensitive polymer under light absorption. For $\gamma_{f} \Delta u \ll 1, \tau$ can be described by

$$
\tau=\tau_{r} \exp \left[-\left(u-u_{r}\right)\left(\gamma_{f} / f_{r}\right)\right] .
$$

This equation and Eqs. (5) and (6) suggest experiments near an energy reference $u_{r}$, say, an energy which leads to the viscoelastic regime of the polymer, much like heating the polymer to near its $T_{g}$, to verify the relationship given by Eq. (7) and measure the parameters $\gamma_{f}$ and $f_{r}$ for different materials. Relaxation processes in glass forming materials are often described by stretched exponentials $\exp \left[-(t / \tau)^{\kappa}\right]$, with $\kappa$ the exponent of the exponential, or similar equations, owing to a distribution of local free volumes available to the probe molecules. That is, Eq. (7) needs to be combined with stretched exponentials to measure relaxation times in polymers under light absorption. It would be interesting to relate $\gamma_{f}$ to the nature of the polymer, $T_{g}$, molecular weight, polymer structure, chain flexibility, mode of attachment of photoactive molecules, liquid crystallinity, and so on, and to measure diffusion times of fluorescent probes in materials, the viscosity of which could be controlled by light.

\section{Anisotropic change of viscosity}

Owing to their highly anisometric polarizability tensor, azo dyes are polarization sensitive, suggesting that photoinduced viscosity changes should also be polarization sensitive. The polarization sensitivity of viscosity could be used to advantage in directional control of viscosity as well as mechanical properties of solid materials in polarized and vectorial light fields for, for example, applications in photomechanics $[27,28]$. When a molecule, oriented at $\Omega(\theta, \varphi)$ in a laboratory coordinate system $(\boldsymbol{x}, \boldsymbol{y}, \boldsymbol{z})$, absorbs a photon of an optical field $\boldsymbol{E}$, say, linearly $\boldsymbol{z}$ polarized, it increases its local free volume and its mobility; if $\chi$ is the angle between the molecular direction $\Omega$ and a probe direction $\boldsymbol{k}$ and $\beta$ is the angle between $\boldsymbol{E}$ and $\boldsymbol{k}$ and averaging about all molecules $N(\Omega)$, with $\psi$ the difference between the azimuthal angles of the molecule and $\boldsymbol{k}$, then

$$
\begin{aligned}
& u(\beta)=\frac{3}{4 \pi} u \int \cos ^{2} \chi \cos ^{2} \theta \sin \theta d \theta d \psi \\
& \cos \chi=\cos \theta \cos \beta+\sin \theta \sin \beta \cos \psi
\end{aligned}
$$

With $P_{2}(\cos \beta)=\left(3 \cos ^{2} \beta-1\right) / 2$ the second-order Legendre polynomial of $\cos \beta$, we have

$$
u(\beta)=u\left[5+4 P_{2}(\cos \beta)\right] / 3 .
$$

Replacing $u$ by $u(\beta)$ wherever it is demonstrates that viscosity changes by light absorption are polarization sensitive indeed. For $\gamma_{f} \Delta u \ll 1$, combining Eqs. (5) and (10) 
yields Eq. (11), and it can be seen from Eqs. (10) and (11), respectively, that the efficiency of the absorbed energy and viscosity change should be in the ratios $1-3$ for the directions $\boldsymbol{k}$ perpendicular to $\boldsymbol{E}(\beta=\pi / 2)$ and at $45^{\circ}\left(\beta=45^{\circ}\right)$ and parallel $(\beta=0)$ to $\boldsymbol{E}$, respectively. Such behavior, e.g., directional photoviscosity, has been observed in $[3,29]$ :

$$
\ln \left[\eta(\beta) / \eta_{r}(\beta)\right]=-\gamma_{f}\left[u(\beta)-u_{r}(\beta)\right] / f_{r} .
$$

\section{COMPARISON WITH EXPERIMENTS}

To study the effect of photoisomerization on viscosity change in detail, experiments along the lines of Fig. 2 must be done; fits to experimental data by Eqs. (5) and (6) would allow for determination of $\gamma_{f}$ and $f_{r}$ and the study of the photochemical properties of polymers in the manner the thermal properties of polymers are studied by the WLF law [19]. Since such studies are unavailable, we will test our theory using data and observations from the literature.

The small decrease of viscosity calculated using Eqs. (1) and (3) in Sec. II A has been observed in a number of polymers. For example, a 20\%-60\% decrease of $\eta$, which indicates a small, i.e., $0.5 \%-1.5 \%$, fractional volume increase by light absorption, has been observed in the literature [30,31]. The materials studied in these references contain photoactive molecules that isomerize by single-step isomerization, such as spiropyranes and symmetrically substituted azobenzenes, which do not continuously cycle between isomers upon light absorption. The trans and cis isomers of these dyes absorb energy in separate regions of the UV-visible spectrum by contrast to DR1. The latter is a push-pull substituted azobenzene which cycles isomerization continuously upon light absorption and must lead to a larger free volume increase in contrast to single-step isomerizing molecules. We will go on to discuss experiments where observation of a viscosity decrease by three to five orders of magnitudes by photoisomerization of azo dyes was reported $[3,4]$.

Comparing the theory to experiments allows for the estimate of $\gamma_{f}$ and for the derivation of a fictitious temperature $T_{f}$ corresponding to athermal viscosity change by light. Let us consider a real sample and estimate $\gamma_{f}$ and $T_{f}$ due to a given light-induced free volume change. We consider a $1-\mu \mathrm{m}$ thick solid film of PMMA and DR1 and a $1-\mathrm{cm}^{2}$ irradiated area of that film, corresponding to an irradiated volume of $10^{-4} \mathrm{~cm}^{3}$, and an absorbed energy dose of $\Delta u \sim 0.49 \mathrm{~J},{ }^{1}$ corresponding to an incident power of $10 \mathrm{~mW}$ for $1 \mathrm{~min}$ of irradiation time. If a $20 \%$ viscosity change, i.e., with $\gamma_{f} \Delta u=$ $5 \times 10^{-3}$, occurs for this sample under the above irradiation conditions, the coefficient of photoexpansion of the polymer is $\gamma_{f} \sim 1.02 \times 10^{-2} \mathrm{~J}^{-1}$. For the estimate of $T_{f}$, we consider a change in fractional free volume of, e.g., $\Delta f=f-f_{r}=$ $5 \times 10^{-3}$, and with $f_{r}=0.024$, which is experimentally measured for PMMA-DR1 [13], and given the coefficient of thermal expansion of PMMA, $\alpha \sim 7 \times 10^{-5} \mathrm{~K}^{-1}$ [32], $\Delta T=$ $f_{r} \gamma_{f} \Delta u / \alpha$ yields $T_{f}\left(T-278 \mathrm{~K} \cong 1.7^{\circ} \mathrm{C}\right)$. Here $T_{f}=T_{g}-$

\footnotetext{
${ }^{1}$ We consider $u_{r}=0$ and to calculate $u$ we use Eq. (1) with $x \sim 0$ and $u=N v \sigma_{T} \phi_{T C} \mathfrak{\Im}$. For DR1-trans, $\sigma_{T}^{532 \mathrm{~nm}} \sim 7.4 \times$ $10^{-17} \mathrm{~cm}^{2}$ molecule ${ }^{-1}, \phi_{T C} \sim 0.11$, and $N=10^{21}$ molecules $\mathrm{cm}^{-3}$.
}

$96.3^{\circ} \mathrm{C}$ means that the polymer must still be in its glassy state. Following the same reasoning for the same sample, under the same light absorption conditions, a 5\% light-induced increase in the fractional free volume, which leads to nearly an order of magnitude decrease in viscosity, yields a hypothetic temperature increase by $17^{\circ} \mathrm{C}$, implying that the polymer must still be in its glassy regime at $T_{f}=T_{g}-81^{\circ} \mathrm{C}$. A small increase in the fractional free volume does not cause a transition of the polymer into the rubbery flow regime.

The expression relating $\Delta T, \alpha$, and $\gamma_{f} \Delta u$ came about by equating the fractional free volume increase $\Delta f$ to light and heat, accepting the hypothetical assumption that all of the absorbed light is transformed into heat. This assumption is used only with the purpose of evaluating the fictitious temperature. So, $\Delta f=\alpha \Delta T=f_{r} \gamma_{f} \Delta u$ for the linear case and $\Delta f=\alpha \Delta T=f_{r}\left(e^{\gamma_{f} \Delta u}-1\right)$ for the general case. These relations should of course be used within reasonable physical intervals of $\Delta T$ and $\Delta u$, with physically sound values of $\alpha$ and $\gamma_{f}$, to avoid divergence of the relation.

Equation (6) shows that if the fractional free volume is increased by $20 \%-30 \%, \eta$ is decreased by three to four orders of magnitude, a situation which corresponds, in the case of the PMMA-DR1 sample, to increasing the irradiation dose to, for example, $50 \mathrm{~mW} \mathrm{~cm}^{-2}$ for an irradiation time of 8-12 min, corresponding to an energy dose absorbed by the sample in the range 20-30 J, a situation which corresponds well to actual experiments, where the viscous motion and poling of the polymer is observed [2-6]. Here $T_{f}$ is equal to $T_{g}-22^{\circ} \mathrm{C}$ and $T_{g}+22{ }^{\circ} \mathrm{C}$ for the $20 \%$ and $30 \%$ increases of $f$, respectively, and it is estimated, for this case, by $\Delta T=\left(e^{\gamma_{f} \Delta u}-1\right)\left(f_{r} / \alpha\right)$, confirming that the spectacular changes of properties of the polymer should occur near $T_{g}$ without light absorption. Light action on polymers increases substantially molecular mobility way below $T_{g}$; this analysis means that, evidently, heating the polymer to near $T_{g}$ must increase the fractional free volume. In addition, $T_{f}$ is indicative of the degree of mobility of the dye upon light absorption and it informs about the extent to which the polymer must be heated, without light absorption, to create the amount of free volume which is athermally created by light. The absorbed photon energy is dissipated into rotational and vibrational motions of the azo dye in a few picoseconds and a fraction of that energy is released into other motions of the local environment of the dye [33]. The free volume created by the photon energy is primarily the result of the inertial transfer of the internal motions of the dye to its local environment upon isomerization.

Reference [5] studied molecular motion, using infrared spectroscopy, when light and heat were applied separately to a polymer sample containing DR1 in the side chain, poly(disperse red 1 acrylate) (pDR1A), a homopolymer, with molecular weight $\sim 5000 \mathrm{~g} / \mathrm{mol}$. It was found that photoisomerization increases the local free volume of the chromophores and corresponds to a fictitious temperature measured from the thermally induced motion of the polymer without light, in agreement with our theoretical developments. While the polymer's $T_{g}$ was $85^{\circ} \mathrm{C}$, photoisomerization led to motion, which would be induced by heating to $240{ }^{\circ} \mathrm{C}$ without photoisomerization, indicating that the polymer has moved athermally from the glassy state to the viscous flow regime. 
A large viscosity decrease in a thin film of an azopolymer by photoisomerization was reported in [3,4]. In [4] it was concluded from the decay of photo-orientation experiments, under light absorption, ignoring orientational randomization of the chromophores by light, that the viscosity of the film was athermally decreased by five orders of magnitude by photoisomerization of a DR1-type azo dye chemically attached to the polymer. It was observed that the higher the light absorption, the larger the decrease of viscosity, in agreement with Eqs. (1) and (2). Indeed, using these equations, this spectacular decrease of viscosity is explained by a large increase in the fractional free volume.

In Ref. [3] the observed large decrease in viscosity by photoisomerization of DR1 attached to the side chain of a pDR1M homopolymer $\left(T_{g} \sim 120^{\circ} \mathrm{C}\right)$ was termed isothermal photo-fluidity. Although the change of viscosity was not measured, motion of the polymer, e.g., flow, along the polarization direction of the actinic light was observed, implying not only a large viscosity change but directional photoviscosity as discussed in Sec. II C. Here too, and as discussed in Ref. [4], the fractional free volume increase must be large and the estimate of $f_{r}$ and $\gamma_{f}$ requires knowledge of the absorbed energy dose and the related change in viscosity of the film. Observation of anisotropic photoviscosity was also reported in Ref. [29] in a similar azopolymer, a PMA-DR1 homopolymer, poly(disperse red 1 methacrylate), very similar to pDR1A. In this reference, anisotropic photoviscous motion, which was observed as polarization sensitive, photoinduced motion of the azopolymer, was made as a function of the energy dose and, interestingly, small and large irradiation doses led to linear and exponential increases in motion, as predicted by Eqs. (5) and (6). Similar observations were made when such a polymer film was exposed to gradients of light intensity, where the polymer, which was initially solid, flowed from the bright to the dark area in the direction of light polarization in symmetric intensity gradients [6]. In this polymer, i.e., PMA DR1, it was calculated that viscosity must be decreased by several orders of magnitude to allow mass flow [7], which occurs in gradient light fields [34].

The first experiment that demonstrated sub- $T_{g}$ enhancement of molecular mobility in photosensitive materials is the so-called photoassisted poling experiment [2]. The sub-
$T_{g}$ light-enhanced mobility of the chromophores was measured by the electro-optic (EO) Pockels coefficient $r_{33}$ and by second harmonic generation ( $\mathrm{SHG}$ ) experiments. It was observed, in a PMMA-DR1 copolymer with a $25 \%$ chromophore concentration $\left(T_{g} \sim 130^{\circ} \mathrm{C}\right)$, that $r_{33}$ increases with the increasing absorbed energy dose at $T_{g}-105^{\circ} \mathrm{C}$, exactly as predicted by the expressions of $u$ and $f$ from Eqs. (1) and (2). Further, $r_{33}$, i.e., poling, is not observable if the polymer is not heated to near $T_{g}$ without photoisomerization, implying that sub- $T_{g}$ molecular motion is frozen without photoisomerization. It was also shown that the extinction coefficient of the chromophore played an important role in the mobility enhancement, as can be seen from Eq. (1). Furthermore, additional EO or SHG experiments can be performed on different azo-dye-containing materials to verify that mobility enhancement scales with the chromophore density $N$ as predicted by Eq. (1) and with $\gamma_{f}$ as predicted by Eq. (2). Clearly, the derived equations not only fill a gap, but also resolve a significant problem and provide a very important tool for studying and developing photosensitive polymers.

\section{CONCLUSION}

In summary, we proposed a model for sub- $T_{g}$ enhancement of molecular mobility in glass forming materials, such as polymers, by light action, which is in good quantitative agreement with experiments. The theory is based on the free volume increase by isomeric rearrangement of photoactive units, and the spectacular decrease of relaxation times and viscosity, in some instances by several decades, from the glassy regime down to the viscous flow regime, is predicted to occur at temperatures way below the glass transition temperature of the material. Two directions could be pursued: first, experimental study of relaxation times and viscosity under light action to better understand the relationship between light absorption and material properties and second, development of materials for directional photoexpansion, e.g., to optimize $\gamma_{f}$.

\section{ACKNOWLEDGMENT}

The author thanks S. Moujdi and S. Refki for help in preparing the graphical figures.
[1] W. Götze and L. Sjögren, Relaxation processes in supercooled liquids, Rep. Prog. Phys. 55, 241 (1992).

[2] Z. Sekkat and M. Dumont, Poling of polymer films by photoisomerisation of azodye chromophores, Mol. Cryst. Liq. Cryst. Sci. Technol. B 2, 359 (1992).

[3] P. Karageorgiev, D. Neher, B. Schulz, B. Stiller, U. Pietsch, M. Giersig, and L. Brehmer, From anisotropic photo-fluidity towards nanomanipulation in the optical near-field, Nat. Mater. 4, 699 (2005).

[4] G. J. Fang, J. E. Maclennan, Y. Yi, M. A. Glaser, M. Farrow, E. Korblova, D. M. Walba, T. E. Furtak, and N. A. Clark, Athermal photo-fluidization of glasses, Nat. Commun. 4, 1521 (2013).

[5] J. Vapaavuori, A. Laventure, C. G. Bazurin, O. L Lebel, and C. Pellerin, Sub-molecular plasticization induced by photons in azobenzene materials, J. Am. Chem. Soc. 137, 13510 (2015).
[6] S. Moujdi, A. Rahmouni, T. Mahfoud, D. V. Nesterenko, M. Halim, and Z. Sekkat, Surface relief gratings in azo-polymers revisited, J. Appl. Phys. 124, 213103 (2018).

[7] Z. Sekkat, Vectorial motion of matter induced by light fueled molecular machines, OSA Continuum 1, 668 (2018).

[8] G. Smets, Photochromic phenomena in the solid phase, Adv. Polym. Sci. 50, 17 (1983), and references therein.

[9] Z. Sekkat, Optical tweezing by photomigration, Appl. Opt. 55, 259 (2016).

[10] V. Teboul, M. Saiddine, and J.-M. Nunzi, IsomerizationInduced Dynamic Heterogeneity in a Glass Former below and above $T_{g}$, Phys. Rev. Lett. 103, 265701 (2009).

[11] G. Tiberio, L. Muccioli, R. Berardi, and C. Zannoni, How does the trans-cis photoisomerization of azobenzene take place in organic solvents? ChemPhysChem 11, 1018 (2010). 
[12] T. Hugel, N. B. Holland, A. Cattani, L. Moroder, M. Seitz, and H. E. Gaub, Single molecule optomechanical cycle, Science 296, 1103 (2002).

[13] Z. Sekkat, G. Kleideiter, and W. Knoll, Optical orientation of azo dye in polymer films at high pressure, J. Opt. Soc. Am. B 18, 1854 (2001).

[14] C. D. Eisenbach, Relation between photochromism of chromophores and free volume theory in bulk polymers, Ber. Bunsen. Phys. Chem. 84, 680 (1980).

[15] M. H. Cohen and D. Turnbull, Molecular transports in liquids and glasses, J. Chem. Phys. 31, 1164 (1959).

[16] A. K. Doolittle and D. B. Doolittle, Studies in Newtonian flow. V. Further verification of the free space viscosity equation, J. Appl. Phys. 28, 901 (1957).

[17] S. K. Kumar, G. Szamel, and J. F. Douglas, Nature of the breakdown in the Stokes-Einstein relationship in a hard sphere fluid, J. Chem. Phys. 124, 214501 (2006).

[18] D. B. Hall, D. D. Deppe, K. E. Hamilton, A. Dhinojwala, and J. M. Torkelson, Probe translational and rotational diffusion in polymers near $\mathrm{T}_{g}$ : Roles of probe size, shape, and secondary bonding in deviations from DebyeStokes-Einstein scaling, J. Non-Cryst. Solids 235-237, 48 (1998).

[19] M. L. Williams, R. F. Landel, and J. D. Ferry, The temperature dependence of relaxation mechanisms in amorphous polymers and other glass-forming liquids, J. Am. Chem. Soc. 77, 3701 (1955).

[20] D. M. Burland, R. D. Miller, and C. A. Walsh, Second order nonlinearity in poled polymer systems, Chem. Rev. 94, 31 (1994).

[21] T. Verbiest, D. M. Burland, M. C. Jurich, V. Y. Lee, R. D. Miller, and W. Volksen, Exceptionally thermally stable polyimides for second-order nonlinear optical applications, Science 268, 1604 (1995).

[22] Y. Q. Shi, C. Zhang, H. Zhang, J. H. Bechtel, L. R. Dalton, B. H. Robinson, and W. H. Steier, Low (sub-1-volt) halfwave voltage polymeric electro-optic modulators achieved by controlling chromophore shape, Science 288, 119 (2000).

[23] H. J. Xu, F. G. Liu, D. L. Elder, L. E. Johnson, Y. de Coene, K. Clays, B. H. Robinson, and L. R. Dalton, Ultrahigh electrooptic coefficients, high index of refraction, and long term stability from Diels-Alder cross-linkable molecular glasses, Chem. Mater. 32, 1408 (2020).

[24] Z. Sekkat, J. Wood, E. F. Aust, W. Knoll, W. Volksen, and R. D. Miller, Light-induced orientation in a high glass transition temperature polyimide with polar azo dyes in the side chain, J. Opt. Soc. Am. B 13, 1713 (1996).

[25] H. Aoki, K. Ishikawa, H. Takezoe, and A. Fukuda, Photoinduced destruction of polar structure in dye-pendant polymers studied by second-harmonic generation, Jpn. J. Appl. Phys. 35, 168 (1996).

[26] Z. Sekkat, A. Knoesen, V. Y. Lee, and R. D. Miller, Influence of the polymer structure on the achievement of polar orientation in high glass transition temperature nonlinear optical polyimides by photo-assisted poling, J. Polym. Sci. B 36, 1669 (1998).

[27] T. J. White, Photomechanical Materials, Composites, and Systems: Wireless Transduction of Light into Work (Wiley, New York, 2017).

[28] B. Zhou, E. Bernhardt, A. Bhuyan, Z. Ghorbanishiadeh, N. Rasmussen, J. Lanska, and M. G. Kuzyk, Theoretical and experimental studies of photomechanical materials, J. Opt. Soc. Am. B 36, 1492 (2019).

[29] H. Ishitobi, M. Tanabe, Z. Sekkat, and S. Kawata, The anisotropic nanomovement of azo-polymers, Opt. Express 15, 652 (2007).

[30] M. Irie, Y. Hirano, S. Hashimoto, and K. Hayashi, Photoresponsive polymers. 2 . Reversible solution viscosity change of polyamides having azobenzene residues in the main chain, Macromolecules 14, 262 (1981).

[31] M. Irie, Y. Hirano, S. Hashimoto, and K. Hayashi, Photoresponsive polymers. Reversible solution viscosity change of poly(methyl methacrylate) having spirobenzopyran side groups, Macromolecules 12, 1176 (1979).

[32] W. D. Drotning and E. P. Roth, Effects of moisture on the thermal expansion of poly(methylmethacrylate), J. Mater. Sci. 24, 3137 (1989).

[33] P. Hamm, S. M. Ohline, and W. Zinth, Vibrational cooling after ultrafast photoisomerization measured by femtosecond infrared spectroscopy, J. Chem. Phys. 106, 519 (1997).

[34] D. Nesterenko, S. Moujdi, S. Hayashi, and Z. Sekkat, Simulation of photochemically induced motion of matter in gradient light fields, J. Appl. Phys. 127, 243106 (2020). 